\title{
Model independent foreground power spectrum estimation using WMAP 5-year data
}

\author{
Tuhin Ghosh, ${ }^{1, *}$ Rajib Saha, ${ }^{1,2,3,4, \dagger}$ Pankaj Jain, ${ }^{4, \ddagger}$ and Tarun Souradeep ${ }^{1, \S}$ \\ ${ }^{1}$ IUCAA, Post Bag 4, Ganeshkhind, Pune-411007, India \\ ${ }^{2}$ Jet Propulsion Laboratory, M/S 169-327, 4800 Oak Grove Drive, Pasadena, California 91109, USA \\ ${ }^{3}$ California Institute of Technology, Pasadena, California 91125, USA \\ ${ }^{4}$ Department of Physics, Indian Institute of Technology, Kanpur, U.P, 208016, India
}

(Received 23 January 2009; published 19 June 2009)

In this paper, we propose and implement on WMAP $5 \mathrm{yr}$ data a model independent approach of foreground power spectrum estimation for multifrequency observations of the $\mathrm{CMB}$ experiments. Recently, a model independent approach of CMB power spectrum estimation was proposed by Saha et al. 2006. This methodology demonstrates that the CMB power spectrum can be reliably estimated solely from WMAP data without assuming any template models for the foreground components. In the current paper, we extend this work to estimate the galactic foreground power spectrum using the WMAP 5 yr maps following a self-contained analysis. We apply the model independent method in harmonic basis to estimate the foreground power spectrum and frequency dependence of combined foregrounds. We also study the behavior of synchrotron spectral index variation over different regions of the sky. We use the full sky Haslam map as an external template to increase the degrees of freedom, while computing the synchrotron spectral index over the frequency range from $408 \mathrm{MHz}$ to $94 \mathrm{GHz}$. We compare our results with those obtained from maximum entropy method foreground maps, which are formed in pixel space. We find that relative to our model independent estimates maximum entropy method maps overestimate the foreground power close to galactic plane and underestimates it at high latitudes.

DOI: 10.1103/PhysRevD.79.123011

PACS numbers: $98.70 . V c, 98.80 . E s$

\section{INTRODUCTION}

The study of galactic foreground emission in the range from few $\mathrm{MHz}$ to few hundreds of $\mathrm{GHz}$ is very important for $\mathrm{CMB}$ observations. Characterizing the foreground emission unravels the galactic physics. Its understanding leads to more reliable removal of foreground contamination from the CMB temperature or polarization anisotropy maps. Diffuse galactic foregrounds consist of three main components : dust, free-free, and synchrotron emission. Recent literature also report evidence of other possible contaminating components like spinning dust, hard synchrotron, and galactic haze at WMAP frequencies [1-5]. It is important to measure the galactic foregrounds components and their spectral behavior unbiased by prior expectations. The physical mechanism and spectral behavior of three main foreground components are summarized below:

(i) Synchrotron emission arises when an electron moves at a relativistic velocity along a magnetic field line. In terms of antenna temperature the frequency dependence of synchrotron emission can be written as

$$
T_{s} \propto \nu^{\beta_{s}},
$$

where the spectral index $\beta_{s}$ varies across the sky. Based on the WMAP data, the WMAP team esti-

\footnotetext{
*tuhin@iucaa.ernet.in

†rajib@caltech.edu

*pkjain@iitk.ac.in

\$tarun@iucaa.ernet.in
}

mated $\beta_{s} \simeq-3.5$ at high latitudes and $\beta_{s} \simeq-2.5$ close to star forming regions near the galactic plane.

(ii) Free-free emission arises when free electrons passing through the hot interstellar medium are deflected and slowed down by ionized atoms, mostly protons. The change of kinetic energy due to deflection is converted into free-free emission. The frequency dependence in terms of antenna temperature can be written as

$$
T_{f} \propto \nu^{\beta_{f}},
$$

where the index $\beta_{f}$ is flatter compared to $\beta_{s}$ and can be approximated as $\beta_{f}=-2.14$ in the WMAP frequency range.

(iii) Dust emission occurs when dust grains in the interstellar medium heated by the photon flux from the stars seek thermal equilibrium by emitting in the infrared or far infrared range of the spectrum. Dust emission can be well modeled by grey body spectrum, and the frequency dependence is given by

$$
T_{d} \propto \frac{\nu^{\beta_{d}+1}}{\exp \left(h \nu / K T_{\text {dust }}\right)-1} \propto \nu^{\beta_{d}} \quad\left(h \nu \ll K T_{\text {dust }}\right) .
$$

Different techniques, such as the maximum entropy method (MEM) $[2,6,7]$ and correlated component analysis [8], have been studied in the literature for modelling individual foreground components in pixel space [23,26-28]. However, all these methods require foreground models in 
terms of templates, e.g., $408 \mathrm{MHz}$ all sky synchrotron emission map (Haslam et al., 1982) $[9,10]$, dust map at $94 \mathrm{GHz}$ by Finkbeiner, Davis, and Schlegel (FDS99) [25], SFD 100 micron dust map [11], and Finkbeiner Halpha maps [4]. While modeling foreground components these methods rely on extrapolation of the external templates to WMAP frequencies. There is always an uncertainty in extrapolating a low frequency or high frequency template to WMAP frequencies [12]. In this paper, we discuss a model independent estimation of foreground behavior without using any information from external templates.

A model independent foreground removal method from the multifrequency $\mathrm{CMB}$ data was first proposed by Tegmark and Efstathiou, 1996 [13], and was implemented on the WMAP 1 year data by Tegmark et al. 2003 [14]. This method was extended by [15-17,29] to estimate CMB power spectrum by cross correlating cleaned maps with independent detector noise. We extend this method to get the spatial and spectral distributions of diffuse foreground power spectrum. The main advantage of this method is that it does not require extrapolation of foreground templates from outside frequency or fitting any foreground template. It is based only on the fact that CMB anisotropy is independent of frequency in the thermodynamic temperature unit, whereas foregrounds have a frequency dependence. Amongst other advantages, it is computationally fast and unlike MEM or correlated component analysis our composite foreground maps are not limited by the lowest resolution frequency band. In this paper, we estimate the power spectrum of the composite emission due to all diffuse foreground components in a self-contained manner using WMAP data only. However, we do not address the problem of extracting the power spectrum due to individual foreground components. By extending the method we provide a map of the variation of the synchrotron spectral index over different regions of the sky.

The rest of the paper is organized as follows.: Section II briefly describes the model independent CMB power spectrum estimation methodology. Section III describes in detail the model independent foreground power spectrum estimation method. Section IV is dedicated to the estimation of synchrotron spectral index over different regions of the sky. Finally, we conclude in Sec. V.

\section{REVIEW OF THE CMB POWER SPECTRUM ESTIMATION}

The basic assumption of model independent CMB power spectrum estimation is that $\mathrm{CMB}$ contributes equally in all frequency channels (in terms of thermodynamic temperature). The temperature anisotropy at a frequency channel $i$ can be written as

$$
\Delta T^{i}(\hat{n})=\int\left\{\Delta T^{C}(\hat{n})+\Delta T_{i}^{F}(\hat{n})\right\} B_{i}\left(\hat{n} . \hat{n}^{\prime}\right) d \hat{n}^{\prime}+\Delta T_{i}^{N}(\hat{n}),
$$

where $\Delta T^{C}(\hat{n})$ and $\Delta T_{i}^{F}(\hat{n})$ are, respectively, the CMB and foreground components, and $\Delta T_{i}^{N}(\hat{n})$ is the noise component. Here, $B_{i}\left(\hat{n} . \hat{n}^{\prime}\right)$ is the circularly symmetric beam denoting the smoothing of the map due to the finite resolution of the detector. The spherical harmonic transform of the map at the frequency channel $i$ can be written as

$$
a_{l m}^{i}=B_{l}^{i} a_{l m}^{C}+B_{l}^{i} a_{l m}^{F}(i)+a_{l m}^{N}(i),
$$

where the index $i$ runs from 1 to 5 for five frequency bands of WMAP. In our analysis, we perform foreground removal in harmonic space and separately over different regions of the sky. We first partition the entire sky in nine different regions according to their level of foreground contamination as described in $[14,17]$ and then perform foreground removal for each region iteratively. Below we briefly describe the basic algorithm of the procedure.

We define a cleaned map as a sum of linearly weighted 5 WMAP maps as follows:

$$
a_{l m}^{\text {clean }}=\sum_{i=1}^{5} W_{l}^{i} \frac{a_{l m}^{i}}{B_{l}^{i}}
$$

where the weight factor depends on the frequency $i$ as well as on the multipole $l$. Since the frequency channels of WMAP are of different resolution, we deconvolve each map by the corresponding beam $B_{l}^{i}$ prior to linear combination. The power spectrum of the cleaned map is given by

$$
\begin{aligned}
C_{l}^{\text {clean }} & =\frac{1}{2 l+1} \sum_{m=-l}^{l} a_{l m}^{\text {clean }} a_{l m}^{\text {clean } *} . \\
& =\frac{1}{2 l+1} \sum_{m=-l}^{l} \sum_{i=1}^{5} W_{l}^{i} \frac{a_{l m}^{i}}{B_{l}^{i}} \sum_{j=1}^{5} W_{l}^{j} \frac{a_{l m}^{j *}}{B_{l}^{j}} .
\end{aligned}
$$

The above equation can be simplified and written as a matrix equation as

$$
\begin{aligned}
C_{l}^{\text {clean }} & =\mathbf{W}_{l} \mathbf{C}_{l} \mathbf{W}_{l}^{T}=\mathbf{W}_{l}\left(\mathbf{C}_{l}^{S}+\mathbf{C}_{l}^{R}\right) \mathbf{W}_{l}^{T} \\
& =C_{l}^{S} \mathbf{W}_{l} \mathbf{e}^{T} \mathbf{W}_{l}^{T}+\mathbf{W}_{l} \mathbf{C}_{l}^{R} \mathbf{W}_{l}^{T},
\end{aligned}
$$

where $\mathbf{e}=(1,1, \ldots, 1)$ is a column vector of five ones,

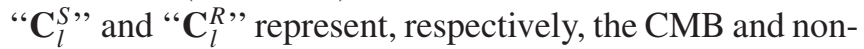
$\mathrm{CMB}$ (i.e., foreground plus detector noise) covariance matrices. $C_{l}^{S}$ is the CMB power spectrum. To preserve the CMB power spectrum in the cleaned map we impose the condition $\mathbf{W}_{l} \mathbf{e}=\mathbf{e}^{T} \mathbf{W}_{l}^{T}=1$. Hence, we can reexpress the above equation as follows:

$$
C_{l}^{\text {clean }}=C_{l}^{S}+\mathbf{W}_{l} \mathbf{C}_{l}^{R} \mathbf{W}_{l}^{T} .
$$

Since in the above equation, CMB power spectrum remains independent of weights, minimizing $C_{l}^{\text {clean }}$ minimizes the combined contamination coming from foregrounds and the detector noise without altering the $\mathrm{CMB}$ power. Minimization of $C_{l}^{\text {clean }}=\mathbf{W}_{l} \mathbf{C}_{l} \mathbf{W}_{l}^{T}$ with the condition that $\mathbf{W}_{l} \mathbf{e}=\mathbf{e}^{T} \mathbf{W}_{l}^{T}=1$ is a standard Lagrangian multiplier problem and has a well-known solution, 


$$
\mathbf{W}_{l}^{T}=\frac{\mathbf{C}_{l}^{-1} \mathbf{e}}{\mathbf{e}^{T} \mathbf{C}_{l}^{-1} \mathbf{e}}
$$

Putting this $\mathbf{W}_{l}^{T}$ back in Eq. (5), we get the cleaned CMB map. The cleaned map in Fourier space can be written as

$$
a_{l m}^{\text {clean }}(i)=a_{l m}^{C}(i)+a_{l m}^{R N}(i),
$$

where "RN" denotes the residual noise in the cleaned map.

\section{ESTIMATION OF FOREGROUND POWER SPECTRUM}

The WMAP satellite has 10 differential assemblies distributed over five different frequencies ranging from $23 \mathrm{GHz}$ to $94 \mathrm{GHz}$. The $\mathrm{K}$ and $\mathrm{Ka}$ bands have one differential assembly (DA) each. The Q and V bands have two DA's each. The two Q-band DA's are labeled Q1 and Q2. Similarly, the V band DA's are labeled V1 and V2. The W band has four DA's, namely, W1, W2, W3, and W4. For the W band, we simply average the pairs of DA's in the W band to form 6 DA maps-W12, W13, W14, W23, W24, W34 out of 4 DA's of the W band. In our analysis, we smooth all the $5 \mathrm{yr}$ WMAP maps to a common beam resolution of $1^{\circ}$. To estimate the cleaned CMB maps, we linearly combine sets of 4 DA's corresponding to different frequencies as described in Sec II and in detail in $[15,17]$. The various different 4-channel combination and 3-channel combination cleaned maps that can be obtained are listed in Table I. Figure 1 shows the comparison between the average of 48 clean maps and WMAP5 ILC map both full sky and outside the KQ85 mask. The right bottom panel of Fig. 1 shows that the difference between ILC and our clean maps is relatively smaller outside the KQ85 mask.
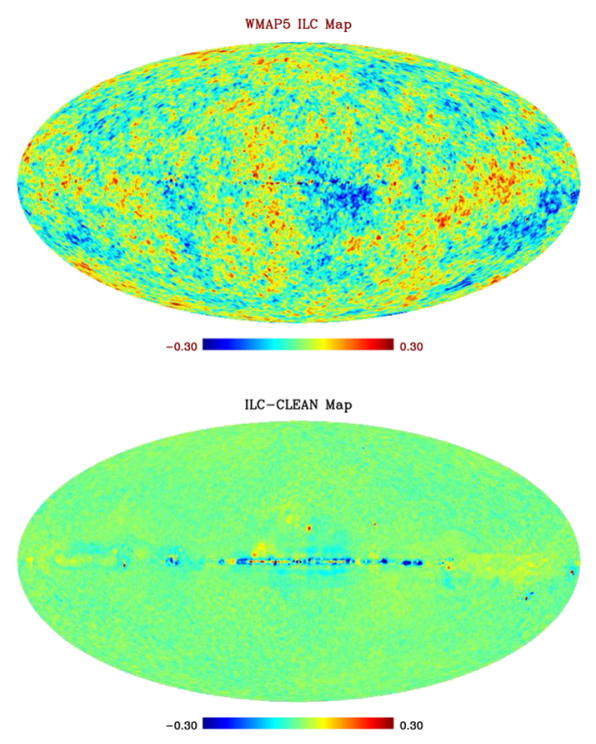

The spherical harmonic transform of the WMAP maps at each frequency channel referred by index $i$, smoothed at $1^{\circ}$ beam resolution can be written as

$$
a_{l m}^{\text {Total }}(i)=B_{l} a_{l m}^{C}+B_{l} a_{l m}^{F}(i)+a_{l m}^{N}(i)
$$

The $1^{\circ}$ smooth cleaned map can be written as

$$
a_{l m}^{\text {clean }}=B_{l} a_{l m}^{C}+B_{l} a_{l m}^{R N},
$$

where " $R N$ " denotes the residual noise in the single cleaned map obtained by our model independent analysis, and $B_{l}$ is the Fourier transform of the beam at $1^{\circ}$ resolution. To obtain the foreground power spectrum, we subtract the cleaned maps from the DA maps obtained as described earlier. The spherical harmonic transform of Di's (see Table II), which is obtained after subtracting the cleaned CMB maps at each frequency channel can now be written as

$$
\begin{aligned}
a_{l m}^{\text {Total }}(i)-a_{l m}^{\text {clean }} & =B_{l} a_{l m}^{F}(i)+\left(a_{l m}^{N}(i)-B_{l} a_{l m}^{R N}\right) . \\
& =B_{l} a_{l m}^{F}(i)+a_{l m}^{N^{\prime}}(i) .
\end{aligned}
$$

To estimate the foreground power spectrum at $1^{\circ}$ beam resolution, we remove the noise bias by cross correlating pairs of CMB free maps, which have no common DA/ detector in the cleaned maps involved. Assuming that the cross correlation between the foreground and detector noise and between the noise corresponding to two independent detectors is negligible, we can write the $1^{\circ}$ beam smoothed foreground power spectrum $C_{l}^{F}$ as

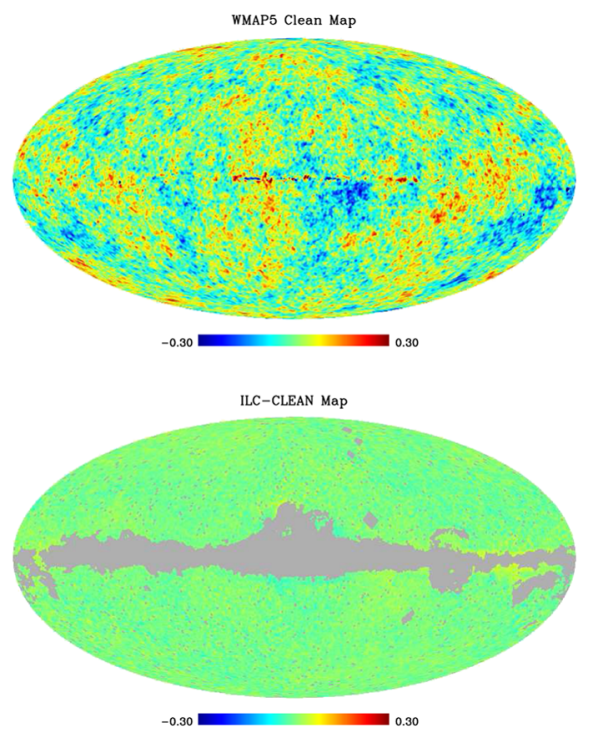

FIG. 1 (color online). The top left panel shows the Internal Linear Combination Map obtained by the WMAP Team. The top right panel shows the average of 48 clean maps obtained by the model independent method. The bottom left and right panel show the difference between the ILC and MI clean map over the full sky and outside the KQ85 mask, respectively. 
TABLE I. The table shows 48 different combinations of the DA maps used in our 4-channel cleaning method and lists the 24 possible combinations in the 3 -channel cleaning method $[15,17]$.

\begin{tabular}{ll}
\hline \hline 4-channel combinations $\left(n_{c}=4\right)$ & \\
\hline$(\mathrm{K}, \mathrm{KA})+\mathrm{Q} 1+\mathrm{V} 1+\mathrm{W} 12=(\mathrm{C} 1, \mathrm{CA} 1)$ & $(\mathrm{K}, \mathrm{KA})+\mathrm{Q} 1+\mathrm{V} 2+\mathrm{W} 12=(\mathrm{C} 13, \mathrm{CA} 13)$ \\
$(\mathrm{K}, \mathrm{KA})+\mathrm{Q} 1+\mathrm{V} 1+\mathrm{W} 13=(\mathrm{C} 2, \mathrm{CA} 2)$ & $(\mathrm{K}, \mathrm{KA})+\mathrm{Q} 1+\mathrm{V} 2+\mathrm{W} 13=(\mathrm{C} 14, \mathrm{CA} 14)$ \\
$(\mathrm{K}, \mathrm{KA})+\mathrm{Q} 1+\mathrm{V} 1+\mathrm{W} 14=(\mathrm{C} 3, \mathrm{CA})$ & $(\mathrm{K}, \mathrm{KA})+\mathrm{Q} 1+\mathrm{V} 2+\mathrm{W} 14=(\mathrm{C} 15, \mathrm{CA} 15)$ \\
$(\mathrm{K}, \mathrm{KA})+\mathrm{Q} 1+\mathrm{V} 1+\mathrm{W} 23=(\mathrm{C} 4, \mathrm{CA} 4)$ & $(\mathrm{K}, \mathrm{KA})+\mathrm{Q} 1+\mathrm{V} 2+\mathrm{W} 23=(\mathrm{C} 16, \mathrm{CA} 16)$ \\
$(\mathrm{K}, \mathrm{KA})+\mathrm{Q} 1+\mathrm{V} 1+\mathrm{W} 24=(\mathrm{C} 5, \mathrm{CA} 5)$ & $(\mathrm{K}, \mathrm{KA})+\mathrm{Q} 1+\mathrm{V} 2+\mathrm{W} 24=(\mathrm{C} 17, \mathrm{CA} 17)$ \\
$(\mathrm{K}, \mathrm{KA})+\mathrm{Q} 1+\mathrm{V} 1+\mathrm{W} 34=(\mathrm{C} 6, \mathrm{CA})$ & $(\mathrm{K}, \mathrm{KA})+\mathrm{Q} 1+\mathrm{V} 2+\mathrm{W} 34=(\mathrm{C} 18, \mathrm{CA} 18)$ \\
$(\mathrm{K}, \mathrm{KA})+\mathrm{Q} 2+\mathrm{V} 2+\mathrm{W} 12=(\mathrm{C} 7, \mathrm{CA})$ & $(\mathrm{K}, \mathrm{KA})+\mathrm{Q} 2+\mathrm{V} 1+\mathrm{W} 12=(\mathrm{C} 19, \mathrm{CA} 19)$ \\
$(\mathrm{K}, \mathrm{KA})+\mathrm{Q} 2+\mathrm{V} 2+\mathrm{W} 13=(\mathrm{C} 8, \mathrm{CA})$ & $(\mathrm{K}, \mathrm{KA})+\mathrm{Q} 2+\mathrm{V} 1+\mathrm{W} 13=(\mathrm{C} 20, \mathrm{CA} 20)$ \\
$(\mathrm{K}, \mathrm{KA})+\mathrm{Q} 2+\mathrm{V} 2+\mathrm{W} 14=(\mathrm{C} 9, \mathrm{CA})$ & $(\mathrm{K}, \mathrm{KA})+\mathrm{Q} 2+\mathrm{V} 1+\mathrm{W} 14=(\mathrm{C} 21, \mathrm{CA} 21)$ \\
$(\mathrm{K}, \mathrm{KA})+\mathrm{Q} 2+\mathrm{V} 2+\mathrm{W} 23=(\mathrm{C} 10, \mathrm{CA} 10)$ & $(\mathrm{K}, \mathrm{KA})+\mathrm{Q} 2+\mathrm{V} 1+\mathrm{W} 23=(\mathrm{C} 22, \mathrm{CA} 22)$ \\
$(\mathrm{K}, \mathrm{KA})+\mathrm{Q} 2+\mathrm{V} 2+\mathrm{W} 24=(\mathrm{C} 11, \mathrm{CA} 11)$ & $(\mathrm{K}, \mathrm{KA})+\mathrm{Q} 2+\mathrm{V} 1+\mathrm{W} 24=(\mathrm{C} 23, \mathrm{CA} 23)$ \\
$(\mathrm{K}, \mathrm{KA})+\mathrm{Q} 2+\mathrm{V} 2+\mathrm{W} 34=(\mathrm{C} 12, \mathrm{CA} 12)$ & $(\mathrm{K}, \mathrm{KA})+\mathrm{Q} 2+\mathrm{V} 1+\mathrm{W} 34=(\mathrm{C} 24, \mathrm{CA} 24)$
\end{tabular}

3-channel combinations $\left(n_{c}=3\right)$

\begin{tabular}{rr}
\hline $\mathrm{Q} 1+\mathrm{V} 1+\mathrm{W} 12=\mathrm{C} 1$ & $\mathrm{Q} 1+\mathrm{V} 2+\mathrm{W} 12=\mathrm{C} 13$ \\
$\mathrm{Q} 1+\mathrm{V} 1+\mathrm{W} 13=\mathrm{C} 2$ & $\mathrm{Q} 1+\mathrm{V} 2+\mathrm{W} 13=\mathrm{C} 14$ \\
$\mathrm{Q} 1+\mathrm{V} 1+\mathrm{W} 14=\mathrm{C} 3$ & $\mathrm{Q} 1+\mathrm{V} 2+\mathrm{W} 14=\mathrm{C} 15$ \\
$\mathrm{Q} 1+\mathrm{V} 1+\mathrm{W} 23=\mathrm{C} 4$ & $\mathrm{~W} 23+\mathrm{V} 2+\mathrm{W} 24=\mathrm{C} 16$ \\
$\mathrm{Q} 1+\mathrm{V} 1+\mathrm{W} 24=\mathrm{C} 5$ & $\mathrm{Q} 1+\mathrm{V} 2+\mathrm{W} 34=\mathrm{C} 18$ \\
$\mathrm{Q} 1+\mathrm{V} 1+\mathrm{W} 34=\mathrm{C} 6$ & $\mathrm{Q} 2+\mathrm{V} 1+\mathrm{W} 12=\mathrm{C} 19$ \\
$\mathrm{Q} 2+\mathrm{V} 2+\mathrm{W} 12=\mathrm{C} 7$ & $\mathrm{Q} 2+\mathrm{V} 1+\mathrm{W} 13=\mathrm{C} 20$ \\
$\mathrm{Q} 2+\mathrm{V} 2+\mathrm{W} 13=\mathrm{C} 8$ & $\mathrm{Q} 2+\mathrm{V} 1+\mathrm{W} 14=\mathrm{C} 21$ \\
$\mathrm{Q} 2+\mathrm{V} 2+\mathrm{W} 14=\mathrm{C} 9$ & $\mathrm{Q} 2+\mathrm{V} 1+\mathrm{W} 23=\mathrm{C} 22$ \\
$\mathrm{Q} 2+\mathrm{V} 2+\mathrm{W} 23=\mathrm{C} 10$ & $\mathrm{Q} 2+\mathrm{V} 1+\mathrm{W} 24=\mathrm{C} 23$ \\
$\mathrm{Q} 2+\mathrm{V} 2+\mathrm{W} 24=\mathrm{C} 11$ & $\mathrm{Q} 2+\mathrm{V} 1+\mathrm{W} 34=\mathrm{C} 24$ \\
$\mathrm{Q} 2+\mathrm{V} 2+\mathrm{W} 34=\mathrm{C} 12$ &
\end{tabular}

$$
\begin{aligned}
& \left\langle\left(a_{l m}^{\text {Total }}(i)-a_{l m}^{\text {clean }}(i)\right)\left(a_{l m}^{\text {Total } *}(j)-a_{l m}^{\text {clean* } *}(j)\right)\right\rangle \\
& =\left\langle B_{l}^{2} a_{l m}^{F}(i) a_{l m}^{F *}(j)\right\rangle+\left\langle a_{l m}^{N^{\prime}}(i) a_{l m}^{N^{\prime *}}(j)\right\rangle . \\
& =C_{l}^{F} .
\end{aligned}
$$

Here, we explain the steps followed to obtain the foreground power spectrum for the $\mathrm{Q}$ band, where the number of DA's are more than one. Similar steps are also followed for the $\mathrm{V}$ band and $\mathrm{W}$ bands.

(i) We smooth all the WMAP DA maps and the cleaned maps to $1^{\circ}$ beam resolution.

(ii) Take the cleaned map $\mathrm{C} 1$ from 4-channel combinations as given in Table I and subtract it from the Q1 map. And similarly take the cleaned map C12 from 4-channel combinations and subtract it from the Q2

\begin{tabular}{|c|c|c|c|}
\hline \multicolumn{2}{|l|}{ CMB subtracted WMAP Q band } & \multicolumn{2}{|c|}{ Cross combinations } \\
\hline $\mathrm{Q} 1-\mathrm{C}[(\mathrm{K}, \mathrm{KA})+\mathrm{Q} 1+\mathrm{V} 1+\mathrm{W} 12]=(\mathrm{D} 01, \mathrm{DA} 01)$ & $\mathrm{Q} 2-\mathrm{C}[(\mathrm{K}, \mathrm{KA})+\mathrm{Q} 2+\mathrm{V} 2+\mathrm{W} 34]=(\mathrm{D} 13, \mathrm{DA} 13)$ & $\mathrm{D} 01 \otimes \mathrm{DA} 13$ & $\mathrm{DA} 01 \otimes \mathrm{D} 13$ \\
\hline $\mathrm{Q} 1-\mathrm{C}[(\mathrm{K}, \mathrm{KA})+\mathrm{Q} 1+\mathrm{V} 1+\mathrm{W} 13]=(\mathrm{D} 02, \mathrm{DA} 02)$ & $\mathrm{Q} 2-\mathrm{C}[(\mathrm{K}, \mathrm{KA})+\mathrm{Q} 2+\mathrm{V} 2+\mathrm{W} 24]=(\mathrm{D} 14, \mathrm{DA} 14)$ & $\mathrm{D} 02 \otimes \mathrm{DA} 14$ & $\mathrm{DA} 02 \otimes \mathrm{D} 14$ \\
\hline $\mathrm{Q} 1-\mathrm{C}[(\mathrm{K}, \mathrm{KA})+\mathrm{Q} 1+\mathrm{V} 1+\mathrm{W} 14]=(\mathrm{D} 03, \mathrm{DA} 03)$ & $\mathrm{Q} 2-\mathrm{C}[(\mathrm{K}, \mathrm{KA})+\mathrm{Q} 2+\mathrm{V} 2+\mathrm{W} 23]=(\mathrm{D} 15, \mathrm{DA} 15)$ & $\mathrm{D} 03 \otimes \mathrm{DA} 15$ & $\mathrm{DA} 03 \otimes \mathrm{D} 15$ \\
\hline $\mathrm{Q} 1-\mathrm{C}[(\mathrm{K}, \mathrm{KA})+\mathrm{Q} 1+\mathrm{V} 1+\mathrm{W} 23]=(\mathrm{D} 04, \mathrm{DA} 04)$ & $\mathrm{Q} 2-\mathrm{C}[(\mathrm{K}, \mathrm{KA})+\mathrm{Q} 2+\mathrm{V} 2+\mathrm{W} 14]=(\mathrm{D} 16, \mathrm{DA} 16)$ & $\mathrm{D} 04 \otimes \mathrm{DA} 16$ & $\mathrm{DA} 04 \otimes \mathrm{D} 16$ \\
\hline $\mathrm{Q} 1-\mathrm{C}[(\mathrm{K}, \mathrm{KA})+\mathrm{Q} 1+\mathrm{V} 1+\mathrm{W} 24]=(\mathrm{D} 05, \mathrm{DA} 05)$ & $\mathrm{Q} 2-\mathrm{C}[(\mathrm{K}, \mathrm{KA})+\mathrm{Q} 2+\mathrm{V} 2+\mathrm{W} 13]=(\mathrm{D} 17, \mathrm{DA} 17)$ & D05 $\otimes$ DA17 & $\mathrm{DA} 05 \otimes \mathrm{D} 17$ \\
\hline $\mathrm{Q} 1-\mathrm{C}[(\mathrm{K}, \mathrm{KA})+\mathrm{Q} 1+\mathrm{V} 1+\mathrm{W} 34]=(\mathrm{D} 06, \mathrm{DA} 06)$ & $\mathrm{Q} 2-\mathrm{C}[(\mathrm{K}, \mathrm{KA})+\mathrm{Q} 2+\mathrm{V} 2+\mathrm{W} 12]=(\mathrm{D} 18, \mathrm{DA} 18)$ & $\mathrm{D} 06 \otimes \mathrm{DA} 18$ & $\mathrm{DA} 06 \otimes \mathrm{D} 18$ \\
\hline $\mathrm{Q} 1-\mathrm{C}[(\mathrm{K}, \mathrm{KA})+\mathrm{Q} 1+\mathrm{V} 2+\mathrm{W} 12]=(\mathrm{D} 07, \mathrm{DA} 07)$ & $\mathrm{Q} 2-\mathrm{C}[(\mathrm{K}, \mathrm{KA})+\mathrm{Q} 2+\mathrm{V} 1+\mathrm{W} 34]=(\mathrm{D} 19, \mathrm{DA} 19)$ & $\mathrm{D} 07 \otimes \mathrm{DA} 19$ & $\mathrm{DA} 07 \otimes \mathrm{D} 19$ \\
\hline $\mathrm{Q} 1-\mathrm{C}[(\mathrm{K}, \mathrm{KA})+\mathrm{Q} 1+\mathrm{V} 2+\mathrm{W} 13]=(\mathrm{D} 08, \mathrm{DA} 08)$ & $\mathrm{Q} 2-\mathrm{C}[(\mathrm{K}, \mathrm{KA})+\mathrm{Q} 2+\mathrm{V} 1+\mathrm{W} 24]=(\mathrm{D} 20, \mathrm{DA} 20)$ & $\mathrm{D} 08 \otimes \mathrm{DA} 20$ & $\mathrm{DA} 08 \otimes \mathrm{D} 20$ \\
\hline $\mathrm{Q} 1-\mathrm{C}[(\mathrm{K}, \mathrm{KA})+\mathrm{Q} 1+\mathrm{V} 2+\mathrm{W} 14]=(\mathrm{D} 09, \mathrm{DA} 09)$ & $\mathrm{Q} 2-\mathrm{C}[(\mathrm{K}, \mathrm{KA})+\mathrm{Q} 2+\mathrm{V} 1+\mathrm{W} 23]=(\mathrm{D} 21, \mathrm{DA} 21)$ & $\mathrm{D} 09 \otimes \mathrm{DA} 21$ & $\mathrm{DA} 09 \otimes \mathrm{D} 21$ \\
\hline $\mathrm{Q} 1-\mathrm{C}[(\mathrm{K}, \mathrm{KA})+\mathrm{Q} 1+\mathrm{V} 2+\mathrm{W} 23]=(\mathrm{D} 10, \mathrm{DA} 10)$ & $\mathrm{Q} 2-\mathrm{C}[(\mathrm{K}, \mathrm{KA})+\mathrm{Q} 2+\mathrm{V} 1+\mathrm{W} 14]=(\mathrm{D} 22, \mathrm{DA} 22)$ & $\mathrm{D} 10 \otimes \mathrm{DA} 22$ & $\mathrm{DA} 10 \otimes \mathrm{D} 22$ \\
\hline $\mathrm{Q} 1-\mathrm{C}[(\mathrm{K}, \mathrm{KA})+\mathrm{Q} 1+\mathrm{V} 2+\mathrm{W} 24]=(\mathrm{D} 11, \mathrm{DA} 11)$ & $\mathrm{Q} 2-\mathrm{C}[(\mathrm{K}, \mathrm{KA})+\mathrm{Q} 2+\mathrm{V} 1+\mathrm{W} 13]=(\mathrm{D} 23, \mathrm{DA} 23)$ & $\mathrm{D} 11 \otimes \mathrm{DA} 23$ & $\mathrm{DA} 11 \otimes \mathrm{D} 23$ \\
\hline $\mathrm{Q} 1-\mathrm{C}[(\mathrm{K}, \mathrm{KA})+\mathrm{Q} 1+\mathrm{V} 2+\mathrm{W} 34]=(\mathrm{D} 12, \mathrm{DA} 12)$ & $\mathrm{Q} 2-\mathrm{C}[(\mathrm{K}, \mathrm{KA})+\mathrm{Q} 2+\mathrm{V} 1+\mathrm{W} 12]=(\mathrm{D} 24, \mathrm{DA} 24)$ & $\mathrm{D} 12 \otimes \mathrm{DA} 24$ & $\mathrm{DA} 12 \otimes \mathrm{D} 24$ \\
\hline
\end{tabular}

TABLE II. The table shows 48 possible combinations of the CMB subtracted WMAP Q-band maps and 24 cross combinations to get rid of noise. 
map. We label the CMB subtracted maps as Di as given in Table II.

(iii) Cross correlate the D01(Q1-C1) with the D13(Q2C12) as listed in Table II to get rid of the noise term and obtain the foreground power spectrum.

(iv) Repeat the above steps for 24 possible combinations obtained by the 4-channel cleaned maps given in Table II. Take the average of the 24 power spectra to obtain the final foreground power spectrum.

Since the $\mathrm{K}$ and $\mathrm{Ka}$ bands have only one DA, cross correlation is not feasible. In these cases, estimate of the contribution of noise bias is explicitly calculated from the number of observation and sensitivity of the detector and subtracted out. To avoid any kind of mixing, we subtract the 3-channel cleaned map $\mathrm{C} 1$ from, say, the $\mathrm{K}$ band and cross correlated it with another 3-channel cleaned map C12 (where the detector used are not common) subtracted $\mathrm{K}$ map as given in Table III. So, Eq. (9) in the case of one DA will become

$$
\begin{aligned}
& \left\langle\left(a_{l m}^{\text {Total }}(i)-a_{l m}^{\text {clean }}(i)\right)\left(a_{l m}^{\text {Total } *}(j)-a_{l m}^{\text {clean } *}(j)\right)\right\rangle \\
& \quad=\left\langle\left(B_{l}^{2} a_{l m}^{F}(i) a_{l m}^{F *}(j)\right)\right\rangle+\left\langle\left(a_{l m}^{N}(i) a_{l m}^{N *}(j)\right)\right\rangle . \\
& \quad=C_{l}^{F}+C_{l}^{N} .
\end{aligned}
$$

The second term $C_{l}^{N}$ on the right-hand side of Eq. (10) can easily be estimated using the relation

$$
C_{l}^{N}=\frac{B_{l}^{2}\left(1^{\circ}\right)}{B_{l}^{2}(K)} \frac{4 \pi}{N_{\mathrm{pix}}^{2}} \sum_{i=1}^{N_{\mathrm{pix}}} \frac{\sigma_{i}^{2}}{N_{\mathrm{obs}}} .
$$

By substituting the value of $C_{l}^{N}$ back into Eq. (10) and subtracting it from the left-hand side gives the $1^{\circ}$ beam smooth foreground power spectrum $\left(C_{l}^{F}\right)$. In general, foreground power spectra are expressed in terms of antenna temperature, which can easily be converted from thermodynamic temperature using Table IV. Henceforth, all the results and plots of $C_{l}^{F}$ are expressed in terms of antenna
TABLE IV. Conversion factor from thermodynamic to antenna temperature [24]where $\Delta T_{A}=g(\nu) \Delta T ; g(\nu)=\left[x^{2} e^{x} /\left(e^{x}-\right.\right.$ $\left.1)^{2}\right] ; x=h \nu / k_{B} T_{0} ; T_{0}=2.725 \mathrm{~K}$.

\begin{tabular}{lccc}
\hline \hline $\begin{array}{l}\text { Frequency } \\
\text { (in GHz) }\end{array}$ & $\begin{array}{c}\text { Conversion factor } \\
g(\nu)\end{array}$ & $\begin{array}{c}\text { Mean } \sigma_{0} \\
\text { (in mK) }\end{array}$ & $\begin{array}{c}\text { Mean FWHM } \\
\text { (in degrees) }\end{array}$ \\
\hline 23 & 0.9867 & 1.436 & 0.807 \\
33 & 0.9723 & 1.470 & 0.624 \\
41 & 0.9581 & 2.197 & 0.4775 \\
61 & 0.9095 & 3.133 & 0.326 \\
94 & 0.8012 & 6.538 & 0.2038 \\
\hline \hline
\end{tabular}

temperature. To get the foreground power spectrum outside the KQ85 mask, we mask the difference map Di's maps with combined KQ85 and WMAP5 point source mask and then smooth to $1^{\circ}$ before cross correlating the Di's where no common DA/detector are present to get rid of noise. Additional smoothing of $1^{\circ}$ is applied outside the KQ85 mask to compare the foreground power spectrum with MEM maps.

We can calculate the root mean square (rms) temperature $\Delta T_{\mathrm{rms}}$ of the foregrounds using the relation

$$
\left(\Delta T_{\mathrm{rms}}\right)^{2}=\sum_{l=2}^{l_{\max }} \frac{2 l+1}{4 \pi} C_{l}^{F},
$$

where $\Delta T_{\mathrm{rms}}$ is expressed in terms of antenna temperature. We obtain the frequency dependent $\Delta T_{\text {rms }}$ for the five WMAP frequencies. We can model the rms foreground power in terms of three major galactic foregrounds-synchrotron, free-free, and dust emission. We fit the $\Delta T_{\mathrm{rms}}$ at the five frequencies to obtain the full sky synchrotron spectral index

$$
\Delta T_{\mathrm{rms}}=A_{s} \nu^{\beta_{s}}+A_{f} \nu^{\beta_{f}}+A_{d} \nu^{\beta_{d}},
$$

where $\beta_{d}=1.8, \beta_{f}=-2.14$ are taken as constant parameters. Fig. 3 shows total foreground emission as a sum

TABLE III. The table shows 24 different combinations of the DA maps for the $\mathrm{K}$ band and 12 cross combinations to get rid of noise. The corresponding set for the Ka band is obtained by replacing $\mathrm{K}$ by $\mathrm{Ka}$.

\begin{tabular}{llr}
\hline \hline CMB subtracted WMAP K maps & & Cross combinations \\
\hline $\mathrm{K}-\mathrm{C}(\mathrm{Q} 1 \mathrm{~V} 1 \mathrm{~W} 12)=\mathrm{D} 01$ & $\mathrm{~K}-\mathrm{C}(\mathrm{Q} 2 \mathrm{~V} 2 \mathrm{~W} 34)=\mathrm{D} 13$ & $\mathrm{D} 01 \otimes \mathrm{D} 13$ \\
$\mathrm{~K}-\mathrm{C}(\mathrm{Q} 1 \mathrm{~V} 1 \mathrm{~W} 13)=\mathrm{D} 02$ & $\mathrm{~K}-\mathrm{C}(\mathrm{Q} 2 \mathrm{~V} 2 \mathrm{~W} 24)=\mathrm{D} 14$ & $\mathrm{D} 02 \otimes \mathrm{D} 14$ \\
$\mathrm{~K}-\mathrm{C}(\mathrm{Q} 1 \mathrm{~V} 1 \mathrm{~W} 14)=\mathrm{D} 03$ & $\mathrm{~K}-\mathrm{C}(\mathrm{Q} 2 \mathrm{~V} 2 \mathrm{~W} 2)=\mathrm{D} 15$ & $\mathrm{D} 03 \otimes \mathrm{D} 15$ \\
$\mathrm{~K}-\mathrm{C}(\mathrm{Q} 1 \mathrm{~V} 1 \mathrm{~W} 23)=\mathrm{D} 04$ & $\mathrm{~K}-\mathrm{C}(\mathrm{Q} 2 \mathrm{~V} 2 \mathrm{~W} 14)=\mathrm{D} 16$ & $\mathrm{D} 04 \otimes \mathrm{D} 16$ \\
$\mathrm{~K}-\mathrm{C}(\mathrm{Q} 1 \mathrm{~V} 1 \mathrm{~W} 24)=\mathrm{D} 05$ & $\mathrm{~K}-\mathrm{C}(\mathrm{Q} 2 \mathrm{~V} 2 \mathrm{~W} 13)=\mathrm{D} 17$ & $\mathrm{D} 05 \otimes \mathrm{D} 17$ \\
$\mathrm{~K}-\mathrm{C}(\mathrm{Q} 1 \mathrm{~V} 1 \mathrm{~W} 34)=\mathrm{D} 06$ & $\mathrm{~K}-\mathrm{C}(\mathrm{Q} 2 \mathrm{~V} 2 \mathrm{~W} 12)=\mathrm{D} 18$ & $\mathrm{D} 06 \otimes \mathrm{D} 18$ \\
$\mathrm{~K}-\mathrm{C}(\mathrm{Q} 1 \mathrm{~V} 2 \mathrm{~W} 12)=\mathrm{D} 07$ & $\mathrm{~K}-\mathrm{C}(\mathrm{Q} 2 \mathrm{~V} 1 \mathrm{~W} 34)=\mathrm{D} 19$ \\
$\mathrm{~K}-\mathrm{C}(\mathrm{Q} 1 \mathrm{~V} 2 \mathrm{~W} 13)=\mathrm{D} 08$ & $\mathrm{~K}-\mathrm{C}(\mathrm{Q} 2 \mathrm{~V} 1 \mathrm{~W} 24)=\mathrm{D} 20$ & $\mathrm{D} 08 \otimes \mathrm{D} 20$ \\
$\mathrm{~K}-\mathrm{C}(\mathrm{Q} 1 \mathrm{~V} 2 \mathrm{~W} 14)=\mathrm{D} 09$ & $\mathrm{~K}-\mathrm{C}(\mathrm{Q} 2 \mathrm{~V} 1 \mathrm{~W} 23)=\mathrm{D} 21$ & $\mathrm{D} 10 \otimes \mathrm{D} 22$ \\
$\mathrm{~K}-\mathrm{C}(\mathrm{Q} 1 \mathrm{~V} 2 \mathrm{~W} 23)=\mathrm{D} 10$ & $\mathrm{~K}-\mathrm{C}(\mathrm{Q} 2 \mathrm{~V} 1 \mathrm{~W} 14)=\mathrm{D} 22$ & $\mathrm{D} 11 \otimes \mathrm{D} 23$ \\
$\mathrm{~K}-\mathrm{C}(\mathrm{Q} 1 \mathrm{~V} 2 \mathrm{~W} 24)=\mathrm{D} 11$ & $\mathrm{~K}-\mathrm{C}(\mathrm{Q} 2 \mathrm{~W} 1 \mathrm{~W} 13)=\mathrm{D} 23$ & $\mathrm{D} 12 \otimes \mathrm{D} 24$ \\
$\mathrm{~K}-\mathrm{C}(\mathrm{Q} 1 \mathrm{~V} 2 \mathrm{~W} 34)=\mathrm{D} 12$ & $\mathrm{~K}-\mathrm{C}(\mathrm{Q} 2 \mathrm{~V} 1 \mathrm{~W} 12)=\mathrm{D} 24$ & \\
\hline \hline
\end{tabular}


of three major foreground components over the full sky. Table V shows the comparison of rms foreground power obtained from the model independent method and the MEM method.

The MEM of foreground estimation employed by the WMAP team uses a model temperature solution based on known galactic components of diffuse emission [2,6,7]. The WMAP team fit the model to data by minimizing the function $H=A+\lambda B$ for each pixel, where $A$ is a standard $\chi^{2}$ fit of the model and $B=\sum_{c} T_{c}(p) \ln \left(T_{c}(p) / P_{c}(p)\right)$. Here, the index $c$ runs over all the galactic components, and $P_{c}(p)$ is a prior model of the spatial distribution of the temperature of the emission component $c$, normalized to the same frequency as $T_{c}$. The parameter $\lambda$ controls the relative weight of $A$ (the data) and $B$ (the prior information). MEM analysis provides output maps of three major galactic components-synchrotron, free-free, and dust for each frequency band of WMAP at an angular resolution of $1^{\circ}$ available at the LAMBDA site. We combine three galactic component maps to obtain a combined MEM foreground map at each frequency band. In this paper, we compare the power spectrum obtained in our model independent analysis to that of the combined MEM foreground emission map at each WMAP frequency. We find that our methods detect marginally lesser foreground power spectrum for the $\mathrm{Q}$ to $\mathrm{W}$ bands as compared to the WMAP MEM method over the full sky. The $\mathrm{K}$ and Ka bands are quite consistent as shown in Fig. 2. The excess power in the
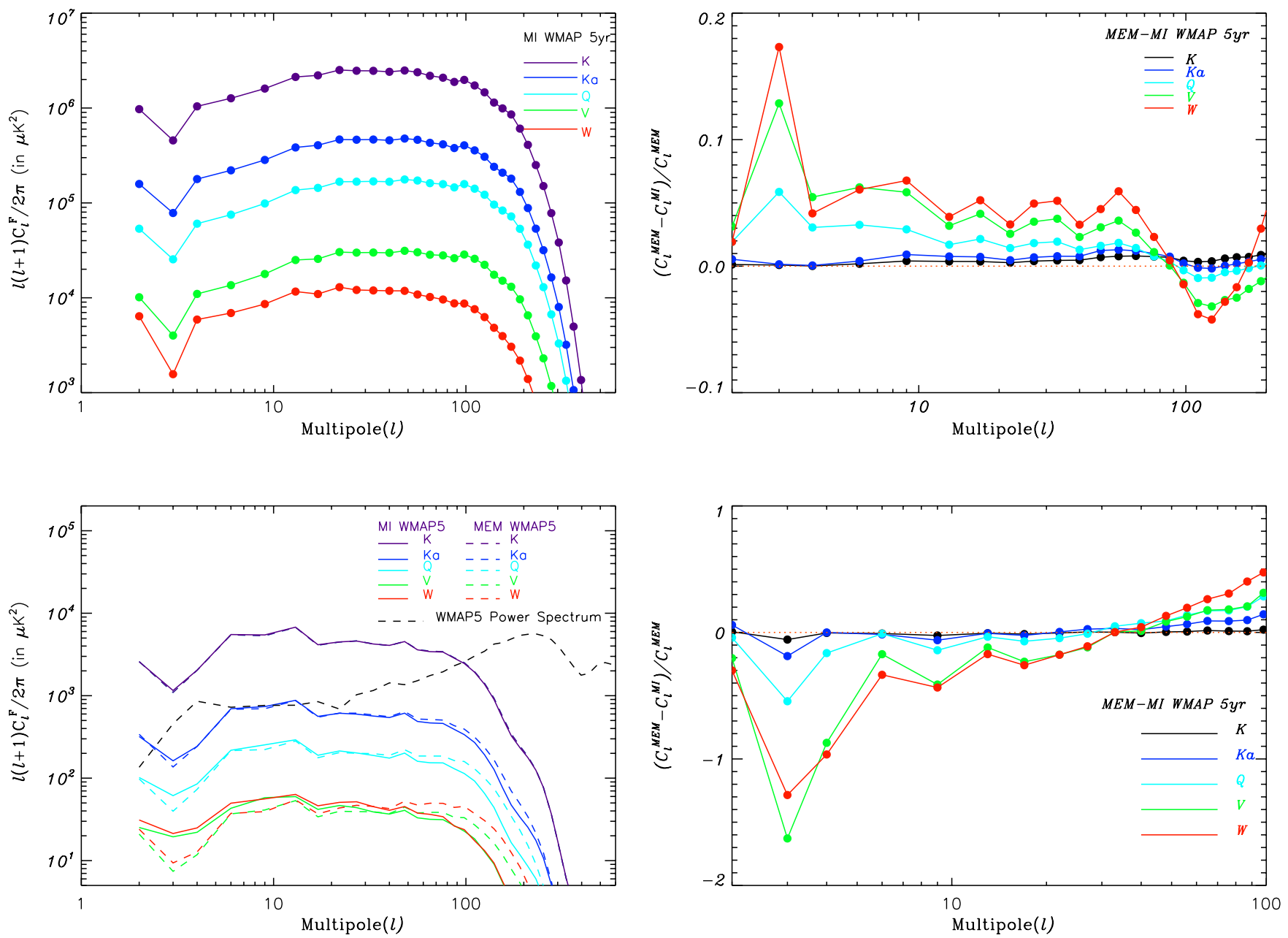

FIG. 2 (color online). Top left panel: The model independent estimate of angular power spectrum for combined foregrounds at WMAP frequency starting from the $\mathrm{K}$ band to the $\mathrm{W}$ band. In this case, $C_{l}^{F}$ is smoothed by a $1^{\circ}$ beam and expressed in terms of antenna temperature. Top right panel: Plot of relative difference in estimated power $\left(C_{l}^{\mathrm{MEM}}-C_{l}^{\mathrm{MI}}\right) / C_{l}^{\mathrm{MEM}}$ with the multipole $l$, where "MI" stands for the foreground power spectrum obtained from model independent analysis. Foreground power spectrum at the K and Ka bands is consistent with the WMAP team. But for the Q, V, and W bands we obtain slightly less power compared to the MEM method. Bottom left panel: The angular power spectrum of combined foregrounds outside the KQ85 mask at WMAP frequencies starting from the $\mathrm{K}$ band to the $\mathrm{W}$ band expressed in antenna temperature. Bottom right panel: Plot of relative power difference $\left(C_{l}^{\mathrm{MEM}}-C_{l}^{\mathrm{MI}}\right) / C_{l}^{\mathrm{MEM}}$ with the multipole $l$ outside KQ85. The plot clearly shows that at low multipoles MEM underestimates the foreground power for a masked sky. 
TABLE V. Comparison of rms foreground power obtained from model independent analysis and the MEM method. The rms foreground power matches closely with the MEM method with a minor deficieny seen in the model independent case.

\begin{tabular}{lcc}
\hline \hline $\begin{array}{l}\text { Frequency } \\
\text { (in GHz) }\end{array}$ & $\begin{array}{c}\text { RMS temperature } \\
\text { using MI analysis } \\
\text { (in mK) }\end{array}$ & $\begin{array}{c}\text { RMS temperature } \\
\text { using MEM maps } \\
\text { (in mK) }\end{array}$ \\
\hline 23 & 2.795 & 2.801 \\
33 & 1.210 & 1.213 \\
41 & 0.731 & 0.736 \\
61 & 0.310 & 0.314 \\
94 & 0.198 & 0.201 \\
\hline \hline
\end{tabular}

WMAP MEM method comes from the low multipoles. Fig. 4 shows the plot of combined foreground maps from $\mathrm{K}$ to $\mathrm{W}$ band obtained by model independent analysis, MEM analysis, and the difference between them over the full sky. To estimate the foreground power outside the KQ85 region, MEM maps are first masked with the KQ85 mask and then smoothed to $1^{\circ}$ beam resolution to get the foreground power spectrum of effective smoothing of $1.414^{\circ}$. We found that the MEM method underestimates the foreground power at low multipoles outside the KQ85 mask. The advantage of the model independent analysis is that the foreground power spectrum is not resolution limited. For each band, we can estimate the foreground power spectrum up to multipoles limited by the beam of that band.

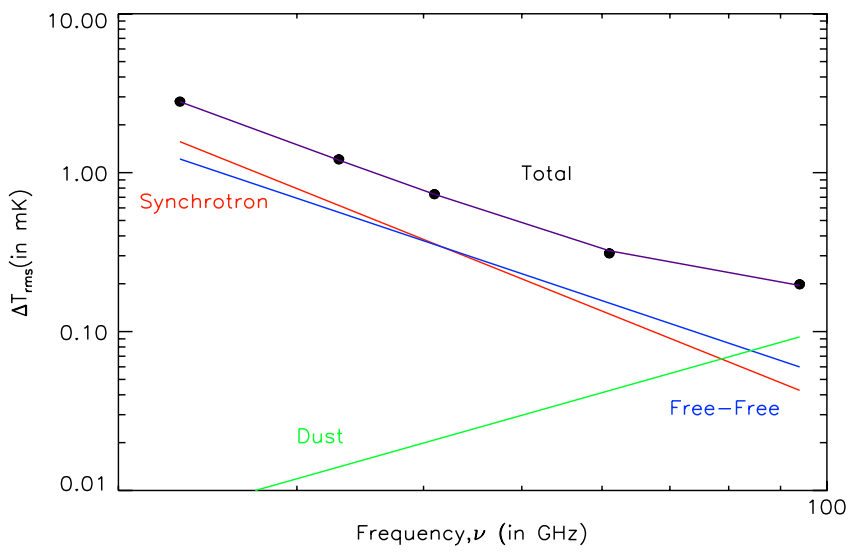

FIG. 3 (color online). The total foreground emission (black dots) spectra obtained using model independent analysis compared to the sum of foreground components (deep blue line connecting black dots) over the full sky. The average synchrotron spectrum from the $\mathrm{K}$ to $\mathrm{W}$ bands is -2.6 assuming the freefree spectral index $\beta_{f}=-2.14$ and dust spectral index $\beta_{d}=$ 1.8 are constant parameters. The average synchrotron spectral index from the K-Ka band and the Ka-Q band are - 3.0 and 2.91 , respectively.

\section{A. Verifying the model independent method using Monte Carlo simulations}

We carry out a set of Monte Carlo simulations to estimate the error on the rms foreground temperature obtained by the model independent analysis. A set of 1000 simulations of CMB maps are made using the HEALPIX software for $N_{\text {side }}=128$ with proper beam width and noise properties for each frequency channel of WMAP $5 \mathrm{yr}$. We choose to downgrade the WMAP maps to $N_{\text {side }}=128$ for our 1000 simulations because the foregrounds are important at low multipoles. We use the publicly available Planck sky model (PSM) version 1.1, to generate the diffuse foreground maps. We estimate 48 (from 4-channel cleaning) and 24 (from 3-channel cleaning) cleaned maps from each realizations as discussed in Sec. II. We subtract them from the input maps and then follow the steps as discussed in Sec. III. Finally, the rms temperature of the foreground from 1000 realizations are recovered and compared with the input values. The mean and variance of recovered foreground rms values are given in Table VI. For 1000 simulations, we get 72000 cleaned maps for $N_{\text {side }}=128$. The simulations shows that our model independent foreground analysis recovers the foreground rms power very well.

While comparing the PSM recovered rms temperature of foregrounds from simulations with that obtained by MEM analysis and our analysis, we note that the PSM foreground templates significantly underestimate the level of galactic contamination. The PSM generated foreground power spectrum for the $\mathrm{K}$ band is much lower than the MEM generated power spectrum. But the difference decreases with the decrease in the level of foreground contamination and for the $\mathrm{W}$ band it matches with the MEM templates. This result has also been noted by other groups [18].

\section{ESTIMATION OF SYNCHROTRON SPECTRAL INDEX OVER DIFFERENT REGIONS OF THE SKY.}

The spectral index of synchrotron emission varies significantly over the sky due to changes in the electron spectrum and density and changes in the magnetic field intensity with the position of the sky $[2,7,9,10,19]$. In the literature, different groups attempt to study the variation of synchrotron spectral index from very low frequency to a few $\mathrm{GHz}$ frequency. Lawson et al., 1987 [20] use the data covering the range from 30 to $1420 \mathrm{MHz}$ to determine the spectral index variation over the northern sky. Jones et al., 2001 [21] determine the spectral index between $408 \mathrm{MHz}$ to $5 \mathrm{GHz}$ in the northern sky. We estimate the spectral index of synchrotron emission over different regions of the sky using the model independent foreground estimation. The regions are defined by the 192 coarser pixels of HEALPIX pixelization at $N_{\text {side }}=4$. Synchrotron spectral index can be calculated easily by knowing the frequency dependence of $\Delta T_{\mathrm{rms}}^{F}$. By definition $\Delta T_{\mathrm{rms}}^{F}$ is given by 


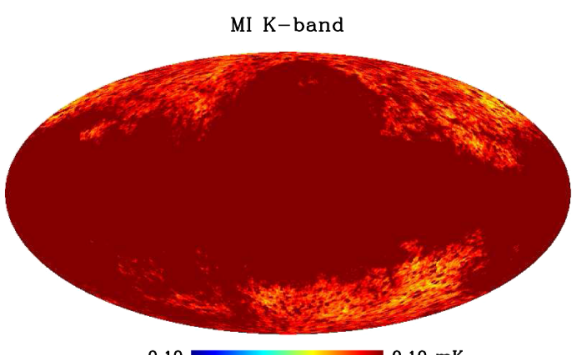

$-0.10 \square 0.10 \mathrm{mK}$

MI Ka-band

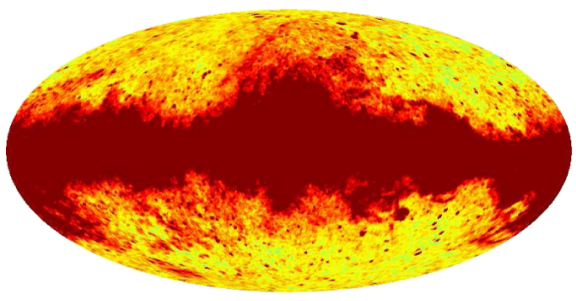

$-0.10 \square 0.10 \mathrm{mK}$

MI Q-band

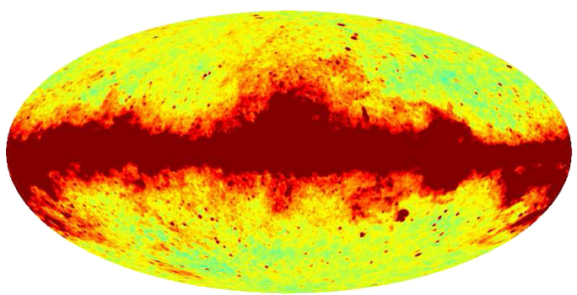

$-0.10=0.10 \mathrm{mk}$

MI V-band

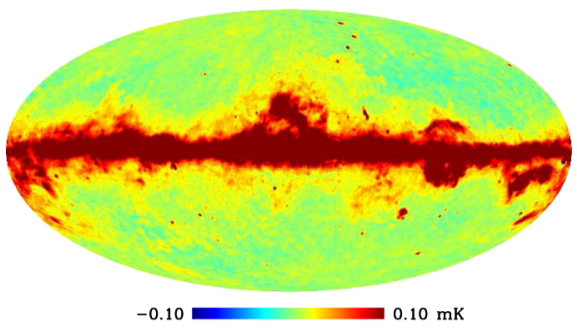

MI W-band

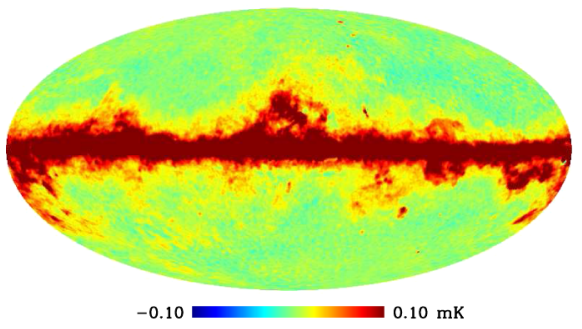

MEM K-band

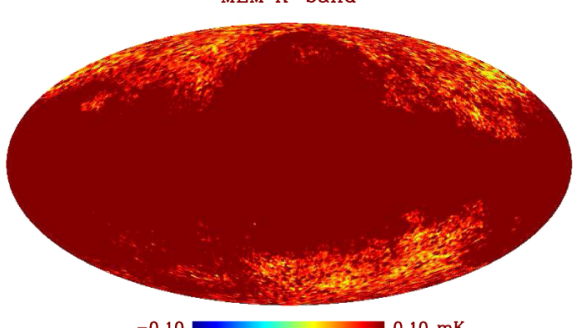

MEM Ka-band

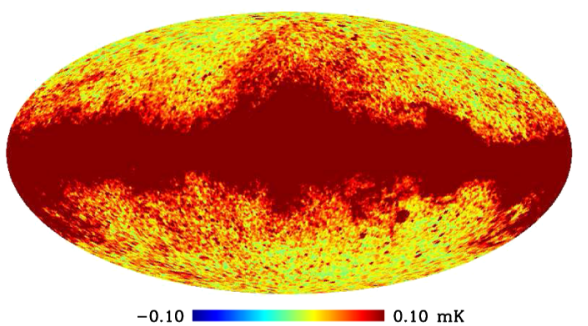

MEM Q-band

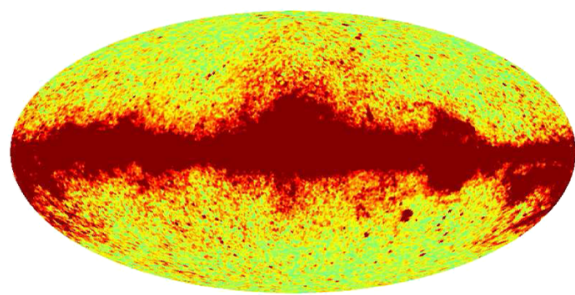

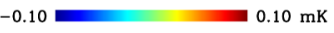

MEM V-band

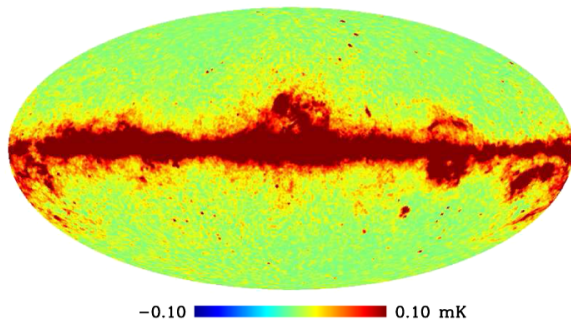

MEM W-band

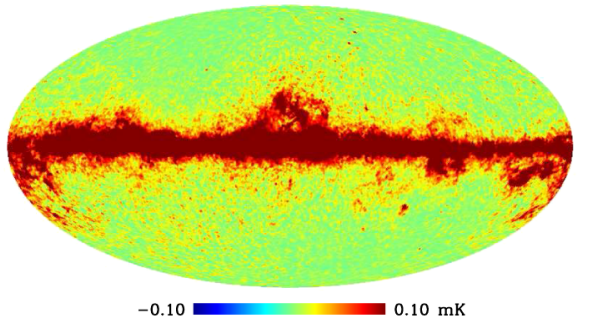

Difference $\mathrm{K}$-band

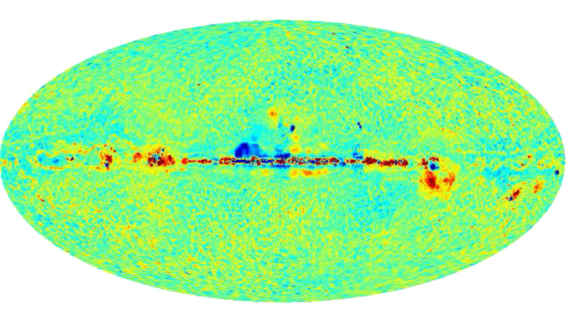

$-0.10=0.10 \mathrm{mK}$

Difference $\mathrm{Ka}-$ band

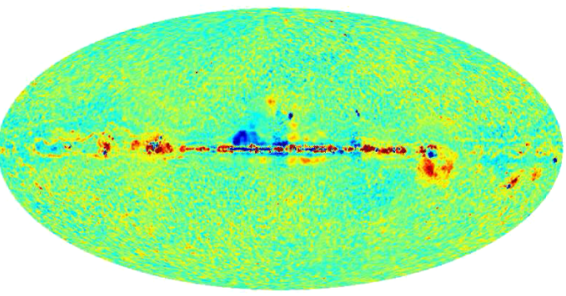

$-0.10=0.10 \mathrm{mK}$

Difference Q-band

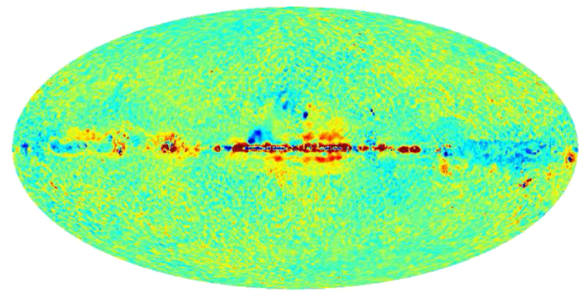

$-0.10=0.10 \mathrm{mK}$

Difference $\mathrm{V}$-band

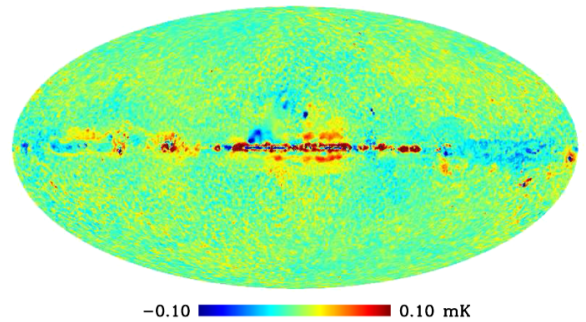

Difference $\mathrm{W}$-band

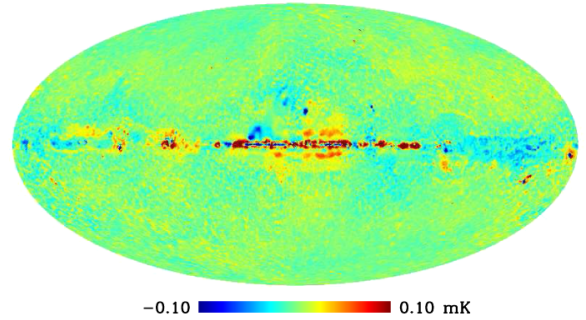

FIG. 4 (color online). The first column is the combined foreground maps for the K, Ka, Q, V, and W maps from top to bottom obtained by model independent analysis. The second column represents the combined foregrounds maps for the K, Ka, Q, V, and W bands obtained by the MEM method, and the third column is MEM minus model independent foreground maps. Model independent foreground maps for $\mathrm{K}$ and $\mathrm{Ka}$ are obtain from 3-channel clean maps, whereas the model independent foreground maps for the Q, V, and $\mathrm{W}$ bands are obtained from 4-channel clean maps. 
TABLE VI. Comparison of input foreground rms temperature (expressed in $\mathrm{mK}$ ) with the recovered foreground rms temperature obtained by Monte Carlo simulations.

\begin{tabular}{lcc}
\hline \hline $\begin{array}{l}\text { Frequency } \\
\text { (in GHz) }\end{array}$ & $\begin{array}{c}\Delta T_{\text {rms }} \text { (in } \mu \mathrm{K} \text { ) input } \\
\text { PSM template }\end{array}$ & $\begin{array}{c}\Delta T_{\text {rms }} \text { (in } \mu \mathrm{K} \text { ) } \\
\text { extracted using our analysis }\end{array}$ \\
\hline 23 & 1440.73 & $1440.64 \pm 3.98$ \\
33 & 628.51 & $627.67 \pm 3.86$ \\
41 & 396.01 & $396.29 \pm 3.94$ \\
61 & 206.85 & $207.23 \pm 3.62$ \\
94 & 181.71 & $182.27 \pm 2.97$ \\
\hline \hline
\end{tabular}

$$
\Delta T_{\mathrm{rms}}^{F}=\left\{\frac{1}{N_{\text {pix }}} \sum_{i=1}^{N_{\text {pix }}}\left\langle\Delta T^{F}(\hat{n})\right\rangle^{2}\right\}^{1 / 2},
$$

where the index " $F$ " represents the combined foreground emission coming from dust emission, free-free emission, synchrotron emission, and $N_{\text {pix }}$ denotes the number of pixels at resolution $N_{\text {side }}=4$ in the given region of the map. $\Delta T_{\text {rms }}^{F}$ is generally expressed in terms of antenna temperature.

Using Eq. (7), the cleaned map in real space can be written as a sum of the CMB map and residual noise as

$$
\Delta T^{\text {clean }}=\Delta T^{C}+\Delta T^{R N} .
$$

Multiplying $\Delta T^{\text {clean }}$ with $1^{\circ}$ resolution beam $B\left(\hat{n} . \hat{n}^{\prime}\right)$ and subtracting it from Eq. (4), we get

$$
\begin{aligned}
\Delta T^{R}(\hat{n}) & =\int\left(\Delta T^{F}(\hat{n})+\Delta T^{R N}(\hat{n})\right) B\left(\hat{n} . \hat{n}^{\prime}\right) d \hat{n}^{\prime}+\Delta T^{N}(\hat{n}) \\
& =\Delta T^{\prime F}(\hat{n})+\Delta T^{N^{\prime}}(\hat{n}),
\end{aligned}
$$

where $\Delta T^{R}(\hat{n})$ denotes the map of the foreground plus the residual noise. The beam smoothed foreground map can be defined as

$$
\Delta T^{\prime F}(\hat{n})=\int \Delta T^{F}(\hat{n}) B\left(\hat{n} \cdot \hat{n}^{\prime}\right) d \hat{n}^{\prime} .
$$

We can define a quantity $\Delta T_{\text {rms }}^{R}$ as

$$
\left(\Delta T_{\mathrm{rms}}^{R}\right)^{2}=\frac{1}{N_{\mathrm{pix}}} \sum_{i=1}^{N_{\mathrm{pix}}}\left\langle\Delta T_{a}^{R}(\hat{n}) \Delta T_{b}^{R}(\hat{n})\right\rangle,
$$

where the indexes " $a$ " and " $b$ " represent the two independent detectors whose noise is uncorrelated. Using the above relation, $\left(\Delta T_{\mathrm{rms}}^{R}\right)^{2}$ can be written as

$$
\begin{aligned}
\left(\Delta T_{\mathrm{rms}}^{R}\right)^{2}= & \frac{1}{N_{\mathrm{pix}}} \sum_{i=1}^{N_{\mathrm{pix}}}\left\langle\Delta T_{a}^{\prime F}(\hat{n}) \Delta T_{b}^{\prime F}(\hat{n})\right\rangle \\
& +\left\langle\Delta T_{a}^{N^{\prime}}(\hat{n}) \Delta T_{b}^{N^{\prime}}(\hat{n})\right\rangle \\
= & \left(\Delta T_{\mathrm{rms}}^{F}\right)^{2}+\left(\Delta T_{\mathrm{rms}}^{N^{\prime}}\right)^{2} .
\end{aligned}
$$

The assumption in the above calculation is that the foreground and noise are uncorrelated. Since the noise for two independent detectors are uncorrelated, the second term vanishes, and we retain only the foreground rms power. The foreground rms temperature for the frequency channel with more than one DA's can be calculated as

$$
\Delta T_{\mathrm{rms}}^{F}=\Delta T_{\mathrm{rms}}^{R}=\left\{\frac{1}{N_{\mathrm{pix}}} \sum_{i=1}^{N_{\mathrm{pix}}}\left\langle\Delta T_{a}^{R}(\hat{n}) \Delta T_{b}^{R}(\hat{n})\right\rangle\right\}^{1 / 2} .
$$

For the $\mathrm{Q}$ to $\mathrm{W}$ bands, where the number of DA's range from two to four, the foreground rms temperature can be calculated using the above equation. For example, to calculate the quantity $\Delta T_{\text {rms }}^{F}$ for the Q band, the steps are as follows:

(i) Smooth all the input WMAP DA maps and the cleaned maps to $1^{\circ}$ beam resolution.

(ii) Subtract the cleaned map C1 obtained by 4 channel combinations from the Q1 band and similarly subtract cleaned map C12 obtained by 4-channel combinations from the Q2 band. The CMB subtracted maps are labeled as Di, and given in Table II.

(iii) Take the cross product of D01(Q1-C1) with the D13 (Q2-C12) over the region of sky defined by a single pixel of $N_{\text {side }}=4$ and take the sum over all the pixels excluding the pixels covered by the WMAP5 point source mask as described in Eq. (14).

(iv) Repeat the above steps for 24 possible combinations produced by 4-channel cleaned maps and take the mean of the resulting 24 power spectrum.

For the $\mathrm{K}$ and $\mathrm{Ka}$ bands where only one DA is present, we subtract 3-channel cleaned maps $\mathrm{C} 1$ from the $\mathrm{K}$ band and subtract 3-channel cleaned map C12 from the K band and take the cross product over the region of sky to get rid of residual noise coming from model independent analysis of CMB power spectrum. Equation (15) for the case of frequency channels with one DA becomes

$$
\left(\Delta T_{\mathrm{rms}}^{R}\right)^{2}=\left(\Delta T_{\mathrm{rms}}^{F}\right)^{2}+\left(\Delta T_{\mathrm{rms}}^{N}\right)^{2},
$$

where $\Delta T_{\mathrm{rms}}^{N}$ can be defined as

$$
\left(\Delta T_{\mathrm{rms}}^{N}\right)^{2}=\frac{1}{N_{\text {pix }}} \sum_{i=1}^{N_{\mathrm{pix}}}\left\langle\Delta T_{a}^{N}(\hat{n}) \Delta T_{b}^{N}(\hat{n})\right\rangle,
$$

which has been estimated using 1000 simulations of noise maps for the $\mathrm{K}$ and $\mathrm{Ka}$ bands smoothed to $1^{\circ}$ beam resolution over the given region of the sky. The rms foreground temperature for the $\mathrm{K}$ and $\mathrm{Ka}$ bands can be calculated using the relation

$$
\Delta T_{\mathrm{rms}}^{F}=\left\{\frac{1}{N_{\mathrm{pix}}} \sum_{i=1}^{N_{\mathrm{pix}}}\left\langle\Delta T_{a}^{R}(\hat{n}) \Delta T_{b}^{R}(\hat{n})\right\rangle\right\}^{1 / 2}-\Delta T_{\mathrm{rms}}^{N} .
$$

The $\Delta T_{\text {rms }}^{F}$ obtained is in terms of thermodynamic temperature, which is converted to antenna temperature using Table IV. For each region, the frequency dependence of $\Delta T_{\mathrm{rms}}^{F}$ is fitted using the relation

$$
\Delta T_{\mathrm{rms}}=A_{s} \nu^{\beta_{s}}+A_{f} \nu^{\beta_{f}}+A_{d} \nu^{\beta_{d}},
$$



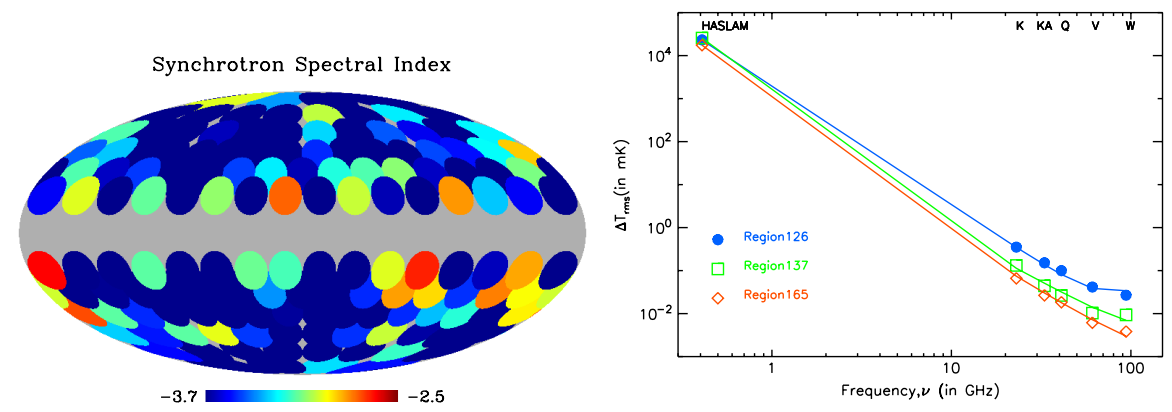

FIG. 5 (color online). Synchrotron spectral index variation over different positions of the sky obtained by model independent foreground analysis. The spectral index behavior clearly shows that $\beta_{s}$ is -3.5 at high latitudes and -2.5 close to the galactic plane, which is consistent with the WMAP team. For example, the fit for 3 regions is shown in the plot. The region 126 (latitude range $\left[10^{\circ}<\ell<29.5^{\circ}\right]$, longitude range $\left[248^{\circ}<b<269^{\circ}\right]$, synchrotron spectral index, $\left.\left[\beta_{s}=-2.82\right]\right)$, region $137\left(-59.1^{\circ}<\ell<\right.$ $\left.-30.7^{\circ}, 23.1^{\circ}<b<44.5^{\circ}, \beta_{s}=-3.70\right)$, region $165\left(-53.3^{\circ}<\ell<-30.3^{\circ}, 248^{\circ}<b<267^{\circ}, \beta_{s}=-3.33\right)$.

where $\beta_{d}=1.8, \beta_{f}=-2.14$ are taken as constant parameters. We fit $\Delta T_{\text {rms }}^{F}$ vs frequency to calculate the synchrotron spectral index at each region of the sky. We calculate the 4 unknown parameters by fitting five frequencies of WMAP at a time, which leads to degeneracy while estimating the synchrotron spectral index. To break the degeneracy, we use the $408 \mathrm{MHz}$ map by Haslam $[9,10]$ as an input template to increase the degrees of freedom while computing the synchrotron spectral index. The Haslam $408 \mathrm{MHz}$ map is an all sky map at a sufficient low frequency, which traces the synchrotron emission at an angular resolution of roughly $1^{\circ}$ provided by LAMBDA. The rms noise level of the Haslam map is $0.5 \mathrm{~K}$ at high latitudes and $0.7 \mathrm{~K}$ at low latitudes. Since the Haslam map is not corrected for noise, the resulting spectral index variation puts an upper limit on the value of average $\beta_{s}$. The spectral index variation at each pixel is shown in Fig. 5 as a sky map where we replace each HEALPIX pixel with a colored circle, which fits inside the pixel. We exclude the galactic region between $\pm 5^{\circ}$ latitude in our analysis. The synchrotron spectral index is found to take values $\beta_{s}=$ -3.5 at high latitudes and $\beta_{s}=-2.5$ close to the galactic plane. The synchrotron spectral index behavior is consistent with the WMAP team [7] and model I of [8].

\section{DISCUSSIONS}

The estimation of foreground power spectrum from WMAP is carried out in a self-contained method without using any extra information at any other frequencies other than those observed by WMAP. This work can be considered as a comprehensive approach that estimates both the CMB power spectrum and the foreground power spectrum simultaneously in a model independent approach. The method described in this paper is unbiased, as we have establish through Monte Carlo simulations. Table VI shows the recovered foreground rms power in the simulations based on the PSM template. Interestingly, we find that the MEM method overestimates the foreground power close to the galactic plane and underestimates the foreground power at high latitudes relative to our estimates. But for the full sky, the mean rms foreground power using the model independent method is close to the MEM method. We find that the average synchrotron spectral index from the $\mathrm{K}$ to $\mathrm{W}$ band is $\beta_{s}=-2.6$ over the full sky. The advantage of this method is that the cross correlations take care of the residual noise that remains after cleaning the map. For the upcoming PLANCK mission, this method of foreground power estimation will be even more promising since there are huge number of cross combinations available due to large number of detectors and greater frequency coverage of PLANCK.

\section{ACKNOWLEDGMENTS}

Computations were carried out on Cetus, the high performance computation facility of IUCAA. Some of the results in this paper used the HEALPIX [22] Package. We acknowledge the use of the Legacy Archive for Microwave Background Data Analysis (LAMBDA). Support for LAMBDA is provided by the NASA office of Space Science. We acknowledge the use of version 1.1 of the Planck reference sky model, prepared by the working group 2 and available at [30]. A portion of the research described in this paper was carried out at the Jet Propulsion Laboratory, California Institute of Technology, under a contract with the National Aeronautics and Space Administration . T. G. thanks the Council of Scientific Research and Industrial Research, India for financial support. 
[1] C. L. Bennett et al. (WMAP Collaboration), Astrophys. J. 583, 1 (2003).

[2] C. L. Bennett et al. (WMAP Collaboration), Astrophys. J. Suppl. Ser. 148, 1 (2003).

[3] A. de Oliveira-Costa, M. Tegmark, L. Page, and S. Bouglin, Astrophys. J. 509, L9 (1998).

[4] D. P. Finkbeiner, Astrophys. J. Suppl. Ser. 146, 407 (2003).

[5] D. P. Finkbeiner, Astrophys. J. 614, 186 (2004).

[6] G. Hinshaw et al. (WMAP Collaboration), Astrophys. J. Suppl. Ser. 170, 288 (2007).

[7] B. Gold et al. (WMAP Collaboration), Astrophys. J. Suppl. Ser. 180, 265 (2009).

[8] A. Bonaldi, S. Ricciardi, S. Leach, F. Stivoli, C. Baccigalupi, and G. de Zotti, Mon. Not. R. Astron. Soc. 382, 1791 (2007).

[9] C. G. T. Haslam, C. J. Salter, H. Stoffel, and W. E. Wilson, Astron. Astrophys. Suppl. Ser. 47, 1 (1982).

[10] C. G. T.Haslam et al., Astron. Astrophys. 100, 209 (1981).

[11] D. J. Schlegel, D. P. Finkbeiner, and M. Davis, Astrophys. J. 500, 525 (1998).

[12] M. Lopez-Corredoira, J. Astrophys. Astron. 28, 101 (2007).

[13] M. Tegmark and G. Efstathiou, Mon. Not. R. Astron. Soc. 281, 1297 (1996).

[14] M. Tegmark, A. de Oliveira-Costa, and A. Hamilton, Phys. Rev. D 68, 123523 (2003).
[15] R. Saha, P. Jain, and T. Souradeep, Astrophys. J. 645, (2006).

[16] T. Souradeep, R. Saha, and P. Jain, New Astron. Rev. 50, 854 (2006).

[17] R. Saha, S. Prunet, P. Jain, and T. Souradeep, Phys. Rev. D 78, 023003 (2008).

[18] S. Ricciardi, New Astron. Rev. 51, 310 (2007).

[19] P. Platania et. al., Astrophys. J. 505, 473 (1998).

[20] K. D. Lawson, M.C. Jayer, J.L. Osborne, and M. Parkinson, Mon. Not. R. Astron. Soc. 225, 307 (1987).

[21] A. W. Jones et al., Mon. Not. R. Astron. Soc. 327, 545 (2001).

[22] K. M. Gorski et al., Astrophys. J. 622, 759 (2005).

[23] C. L. Bennett et al. (WMAP Collaboration), Astrophys. J. Suppl. Ser. 148, 97 (2003).

[24] M. Limon et al., Five Year WMAP Explanatory Supplement, version 3.0, at the LAMBDA website.

[25] D. P. Finkbeiner, M. Davis, and D. J. Schlegel, Astrophys. J. 524, 867 (1999).

[26] J. Delabrouille and J.F. Cardoso, arXiv:astro-ph/ 0702198v2.

[27] S. M. Leach et al., Astron. Astrophys. 491, 597 (2008).

[28] F. R. Bouchet and R. Gispert, New Astron. Rev. 4, 443 (1999).

[29] H. K. Eriksen et al., Astrophys. J. 656, 641 (2007).

[30] www.planck.fr/heading79.html. 